\title{
Spectral Distribution of Transport Operator Arising in Growing Cell Populations
}

\author{
Hongxing Wu, Shenghua Wang, and Dengbin Yuan \\ Department of Mathematics, Shangrao Normal University, Shangrao, Jiangxi 334001, China \\ Correspondence should be addressed to Hongxing Wu; jxsruwhx@163.com
}

Received 27 May 2014; Accepted 12 August 2014; Published 25 August 2014

Academic Editor: Leszek Olszowy

Copyright ( 2014 Hongxing Wu et al. This is an open access article distributed under the Creative Commons Attribution License, which permits unrestricted use, distribution, and reproduction in any medium, provided the original work is properly cited.

\begin{abstract}
Transport equation with partly smooth boundary conditions arising in growing cell populations is studied in $L_{p}(1<p<+\infty)$ space. It is to prove that the transport operator $A_{H}$ generates a $C_{0}$ semigroup and the ninth-order remainder term $R_{9}(t)$ of the Dyson-Phillips expansion of the semigroup is compact, and the spectrum of transport operator $A_{H}$ consists of only finite isolated eigenvalues with finite algebraic multiplicities in a trip $\Gamma_{\omega}$. The main methods rely on theory of linear operators, comparison operators, and resolvent operators approach.
\end{abstract}

\section{Related Knowledge}

In this paper, we are concerned with the following transport equation, which was proposed by Rotenberg in [1]:

$$
\begin{aligned}
\frac{\partial \psi}{\partial t}(\mu, v, t)= & -v \frac{\partial \psi}{\partial \mu}(\mu, v, t)-\sigma(\mu, v) \psi(\mu, v, t) \\
& +\int_{0}^{c} r\left(\mu, v, v^{\prime}\right) \psi\left(\mu, v^{\prime}, t\right) d v^{\prime},
\end{aligned}
$$

with the initial condition and the general biological rule

$$
\begin{gathered}
\psi(\mu, v, 0)=\psi_{0}(\mu, v), \\
\psi^{0}(0, v, t)=H \psi^{a}(a, v, t),
\end{gathered}
$$

where $\mu$ is maturity degree of cells, $\mu \in[0, a], a>0$; the degree of maturation $\mu$ is then defined in the manner that $\mu=0$ at the birth and $\mu=a$ at the death, and their maturation velocity $v, v^{\prime} \in[0, c], c>0, \psi(\mu, v, t)$ describes the number density of cell population as a function of the degree of maturation $\mu$; the maturation velocity $v$ and the time $t, \sigma(\mu, v)$ denote the total transition cross-section while the function $r\left(\mu, v, v^{\prime}\right)$ represents the transition rate at which cells change their velocities from $v$ to $v^{\prime}, \psi(\mu, v, 0)=\psi_{0}(\mu, v)$ is initial condition, $H$ is linear operator in boundary space and is known as transition rule in biology.
It is well known that the streaming operator generates a strongly continuous semigroup $\left(U_{H}(t)_{t \geq 0}\right)$ (see, e.g., [2-5]). If the collision operator is bounded, then the classical perturbation theory (see, [6-8]) shows that the transport operator generates also a strongly continuous semigroup $\left(V_{H}(t)_{t \geq 0}\right)$ (see, e.g., [9]) given by the Dyson-Phillips expansion:

$$
V_{H}(t)=\sum_{j=0}^{n-1} U_{j}(t)+R_{n}(t),
$$

where $U_{0}(t)=U_{H}(t)$ and

$$
U_{i}(t)=\int_{0}^{t} U_{H}(s) K U_{j-1}(t-s) d s, \quad j=1,2, \ldots,
$$

where $K$ represents disturbance operators, and the remainder term is given by

$$
\begin{aligned}
& R_{n}(t)= \sum_{j=n}^{+\infty} U_{j}(t) \\
&=\int_{t_{1}+\cdots+t_{n} \leq t, t_{i} \geq 0} U_{H}\left(t_{1}\right) \\
& \quad \times K U_{H}\left(t_{2}\right) K \cdots U_{H}\left(t_{n}\right) K \\
& \quad \times V_{H}\left(t-t_{1} \cdots-t_{n}\right) d t_{1} \cdots d t_{n} .
\end{aligned}
$$


As is well known, the solution $\psi(t)$ of (1)-(2) exists and is unique for all $\psi_{0} \in D\left(A_{H}\right)$; here $A_{H}$ represents transport operator $A$ with boundary condition $H$.

For the past few years, there are many research works on the spectrum analysis of (1), and some of them had been discussed in [6,10-16]. Jeribi et al. [10] discussed Rotenberg's model of cell population with general compact boundary conditions and proved that the transport semigroup was irreducible, and a spectral decomposition of the solution into an asymptotic term was derived. Latrach and Megdiche [11] discussed the large time behavior of the solution to the Cauchy problem governed by a transport equation with Maxwell compact boundary conditions arising in growing cell population in $L_{1}$ spaces and proved that the remainder term was compact and got

$$
r_{e}\left(V_{H}(t)\right)=r_{e}\left(U_{H}(t)\right) .
$$

Wang and Cheng [13] had proved that the transport operator with compact integral boundary conditions generated a $C_{0}$ semigroup and the existence of the eigenvalues in $L_{p}(1 \leq$ $p<+\infty)$ space in L-R's model. So, it is natural to set the following question: what happens about the transport operator spectral distribution in Rotenberg's model when boundary conditions are partly compact? In this paper, we discuss the transport equation with partly smooth boundary conditions arising in growing cell populations in $L_{p}(1<p<$ $+\infty)$ space. It is to prove that the transport operator generates a $C_{0}$ semigroup and the ninth-order remainder term $R_{9}(t)$ of the Dyson-Phillips expansion of the semigroup is compact, and the spectrum of transport operator consists of only finite isolated eigenvalues with finite algebraic multiplicities in a trip $\Gamma_{\omega}$.

Now, we introduce different notions and notations. Let us first make precisely the functional setting of the problem. Let

$$
X_{p}=L_{p} \quad(1<p<+\infty)([0, a] \times[0, c] ; d \mu d v),
$$

whose norm is

$$
\|\psi\|_{X^{p}}=\left(\int_{0}^{a} \int_{0}^{c}|\psi(\mu, v)| d \mu d v\right)^{1 / p} .
$$

We denote by $X_{p}^{0}, X_{p}^{a}$ the following boundary spaces:

$$
X_{p}^{0}=L_{p} \quad\left(\Gamma_{0} ; v d v\right), \quad X_{p}^{a}=L_{p} \quad\left(\Gamma_{a} ; v d v\right),
$$

where

$$
\Gamma_{0}=\{0\} \times[0, c], \quad \Gamma_{a}=\{a\} \times[0, c] .
$$

In the sequel, $X_{p}^{0}$ and $X_{p}^{a}$ will often be identified with $L_{p}([0, c] ; v d v)$. We define the partial Sobolev space $W_{p}$ as follows:

$$
W_{p}=\left\{\psi \in X_{p}, v \frac{\partial \psi}{\partial \mu} \in X_{p}\right\}
$$

We define the disturbance operators $K$ and streaming operator $T_{H}$ by

$$
\begin{aligned}
(K \psi)(\mu, v) & =\int_{0}^{c} r\left(\mu, v, v^{\prime}\right) \psi\left(\mu, v^{\prime}\right) d v^{\prime}, \\
T_{H} \psi(\mu, v) & =-v \frac{\partial \psi}{\partial \mu}(\mu, v)-\sigma(\mu, v) \psi(\mu, v), \\
D\left(T_{H}\right) & =\left\{\psi \in W_{p} \mid \psi^{0}=H \psi^{a}\right\} .
\end{aligned}
$$

So we may define the transport operator $A_{H}$ by

$$
A_{H}=T_{H}+K, \quad D\left(A_{H}\right)=D\left(T_{H}\right) .
$$

Let $\sigma_{0}$ be the real defined by

$$
\sigma_{0}=\operatorname{ess} \inf \{\sigma(\mu, v),(\mu, v) \in[0, a] \times[0, c]\} .
$$

Consider now the resolvent equation for operator $T_{H}$ :

$$
\left(\lambda I-T_{H}\right) \psi=\varphi,
$$

where $\varphi$ is a given function of $X_{p}$. For $\operatorname{Re} \lambda>-\sigma_{0}$ the solution is formally given by

$$
\begin{aligned}
\psi(\mu, v)= & \psi(0, v) e^{-(1 / v) \int_{0}^{\mu}\left(\lambda+\sigma\left(\mu^{\prime}, v\right)\right) d \mu^{\prime}} \\
& +\frac{1}{v} \int_{0}^{\mu} e^{-(1 / v) \int_{\mu^{\prime}}^{\mu}(\lambda+\sigma(\xi, v)) d \xi} \varphi\left(\mu^{\prime}, v\right) d \mu^{\prime} .
\end{aligned}
$$

Accordingly, for $\mu=a$, we get

$$
\begin{aligned}
\psi(a, v)= & \psi(0, v) e^{-(1 / v) \int_{0}^{a}\left(\lambda+\sigma\left(\mu^{\prime}, v\right)\right) d \mu^{\prime}} \\
& +\frac{1}{v} \int_{0}^{a} e^{-(1 / v) \int_{\mu^{\prime}}^{a}(\lambda+\sigma(\xi, v)) d \xi} \varphi\left(\mu^{\prime}, v\right) d \mu^{\prime}
\end{aligned}
$$

Now, let us define the transport operators $P_{\lambda}, Q_{\lambda}, D_{\lambda}$, and $E_{\lambda}$ by

$$
P_{\lambda}: X_{p}^{0} \longrightarrow X_{p}^{a}
$$

$$
\begin{gathered}
\left(P_{\lambda} g\right)(a, v)=g(0, v) e^{-(1 / v) \int_{0}^{a}\left(\lambda+\sigma\left(\mu^{\prime}, v\right)\right) d \mu^{\prime}}, \\
Q_{\lambda}: X_{p}^{0} \longrightarrow X_{p},
\end{gathered}
$$

$$
\begin{gathered}
\left(Q_{\lambda} g\right)(\mu, v)=g(0, v) e^{-(1 / v) \int_{0}^{\mu}\left(\lambda+\sigma\left(\mu^{\prime}, v\right)\right) d \mu^{\prime}}, \\
D_{\lambda}: X_{p} \longrightarrow X_{p}^{a},
\end{gathered}
$$$$
\left(D_{\lambda} g\right)(a, v)
$$

$$
\begin{aligned}
& =\frac{1}{v} \int_{0}^{a} e^{-(1 / v) \int_{\mu^{\prime}}^{a}(\lambda+\sigma(\xi, v)) d \xi} g\left(\mu^{\prime}, v\right) d \mu^{\prime}, \\
& E_{\lambda}: X_{p} \longrightarrow X_{p}, \\
& \left(E_{\lambda} g\right)(\mu, v) \\
& =\frac{1}{v} \int_{0}^{\mu} e^{-(1 / v) \int_{\mu^{\prime}}^{\mu}(\lambda+\sigma(\xi, v)) d \xi} g\left(\mu^{\prime}, v\right) d \mu^{\prime} .
\end{aligned}
$$


Clearly, for $\lambda$ satisfying $\operatorname{Re} \lambda>-\sigma_{0}$, the operators $P_{\lambda}, Q_{\lambda}$, $D_{\lambda}$, and $E_{\lambda}$ are bounded and positive, and it is not difficult to check that

$$
\begin{gathered}
\left\|D_{\lambda}\right\| \leq 1, \\
\left\|P_{\lambda}\right\| \leq e^{-(a / c)\left(\operatorname{Re} \lambda+\sigma_{0}\right)}, \\
\left\|Q_{\lambda}\right\| \leq\left(\operatorname{Re} \lambda+\sigma_{0}\right)^{-1}, \\
\left\|E_{\lambda}\right\| \leq\left(\operatorname{Re} \lambda+\sigma_{0}\right)^{-1} .
\end{gathered}
$$

Because of (18), we can write

$$
\psi^{a}=P_{\lambda} K \psi^{a}+D_{\lambda} \varphi
$$

Let $\lambda_{0}$ be a real number defined by

$$
\lambda_{0}= \begin{cases}-\sigma_{0}, & \text { if }\|H\| \leq 1, \\ -\sigma_{0}+\frac{c}{a} \log (\|H\|), & \text { if }\|H\|>1 .\end{cases}
$$

Clearly, for $\operatorname{Re} \lambda>-\lambda_{0}$, we get

$$
\psi^{a}=\left(I-P_{\lambda} K\right)^{-1} D_{\lambda} \varphi .
$$

Because of (17) and (23) we get

$$
\psi=Q_{\lambda} K\left(I-P_{\lambda} K\right)^{-1} D_{\lambda} \varphi+E_{\lambda} \varphi .
$$

Accordingly, for $\operatorname{Re} \lambda>-\lambda_{0}$, the resolvent of the operator $T_{H}$ is given by

$$
\left(\lambda I-T_{H}\right)^{-1}=\sum_{n \geq 0} Q_{\lambda} H\left(P_{\lambda} H\right)^{n} D_{\lambda}+E_{\lambda} .
$$

In the following, we assume that $H$ and $K$ satisfy the following:

$\left(\mathrm{O}_{1}\right) H=H_{1}+H_{2}$, where $H_{1}$ is bounded and positive operator and $\mathrm{H}_{2}$ is compact and positive operator;

$\left(\mathrm{O}_{2}\right) K$ is regular operator in $X_{p}$. So it can be approximated in the uniform operator topology by operators; then

$$
\begin{array}{rl}
\int_{0}^{c} & r\left(\mu, v, v^{\prime}\right) \psi\left(\mu, v^{\prime}\right) d v^{\prime} \\
& =\sum_{i \in I} \alpha(\mu) f_{i}(v) \int_{0}^{c} g_{i}\left(v^{\prime}\right) \varphi\left(\mu, v^{\prime}\right) d v^{\prime}
\end{array}
$$

where $\alpha_{i}(\cdot) \in L_{\infty}([0, a], d \mu), f_{i}(\cdot) \in L_{p}([0, c], d v)$, $g_{i}(\cdot) \in L_{q}([0, c], d v),(1 / p)+(1 / q)=1$, and $I$ is finite set.

Indeed, let us first observe that if we replace in the definition of $T_{H}$ the function $\sigma(\mu, v)$ by the real $\sigma_{0}$, we obtain a new streaming operator which we denote by $\overline{T_{H}}$. Arguing as above we can define the operators $\overline{P_{\lambda}}, \overline{Q_{\lambda}}, \overline{D_{\lambda}}$, and $\overline{E_{\lambda}}$, which satisfy, for any $\operatorname{Re} \lambda>\lambda_{0}$,

$$
\left(\lambda I-\overline{T_{H}}\right)^{-1}=\sum_{n \geq 0} \overline{Q_{\lambda}} H\left(\overline{P_{\lambda}} H\right)^{n} \overline{D_{\lambda}}+\overline{E_{\lambda}} .
$$

Lemma 1. For any $t>0, n \in \mathbf{N}$, then

$$
0 \leq R_{n}(t) \leq \overline{R_{n}(t)}
$$

where $R_{n}(t)$ and $\overline{R_{n}(t)}$ denote the $n$th remainder term of the Dyson-Phillips expansion of the semigroup generated by $A_{H}$ and $\overline{A_{H}}$.

Proof. For all $\operatorname{Re} \lambda>\lambda_{0}$, a simple calculation shows that

$$
\begin{array}{ll}
\overline{M_{\lambda}} \geq M_{\lambda}, & \overline{N_{\lambda}} \geq N_{\lambda}, \\
\overline{D_{\lambda}} \geq D_{\lambda}, & \overline{E_{\lambda}} \geq E_{\lambda} .
\end{array}
$$

For (25), (27), and (29) we get

$$
\left(\lambda I-T_{H}\right)^{-1} \leq\left(\lambda I-\overline{T_{H}}\right)^{-1} .
$$

Let $t>0$ be a fixed real; by (30), it is obvious that, for all integer $n \in N$ and all $\psi \in\left(X_{p}\right)_{+}$such that $n / t>\lambda$, we have

$$
0 \leq \frac{n}{t}\left(\frac{n}{t}-T_{H}\right)^{-1} \psi \leq \frac{n}{t}\left(\frac{n}{t}-\overline{T_{H}}\right)^{-1} \psi
$$

Consequently,

$$
\begin{aligned}
0 & \leq \lim _{n \rightarrow+\infty} \frac{n}{t}\left(\frac{n}{t}-T_{H}\right)^{-1} \psi \\
& \leq \lim _{n \rightarrow+\infty} \frac{n}{t}\left(\frac{n}{t}-\overline{T_{H}}\right)^{-1} \psi .
\end{aligned}
$$

Next, in view of the exponential formula for strongly continuous semigroups, we have

$$
0 \leq U_{H}(t) \leq \overline{U_{H}(t)}
$$

where $U_{H}(t)_{t \geq 0}$ and $\overline{U_{H}(t)_{t \geq 0}}$ denote the strongly continuous semigroup generated by $T_{H}$ and $\overline{T_{H}}$. The positivity of $K$ and (30) imply that

$$
\begin{aligned}
0 & \leq\left[\left(\lambda I-T_{H}\right)^{-1} K\right]^{n}\left(\lambda I-T_{H}\right) \\
& \leq\left[\left(\lambda I-\overline{T_{H}}\right)^{-1} K\right]^{n}\left(\lambda I-\overline{T_{H}}\right) .
\end{aligned}
$$

Now, we define transport operator $\overline{A_{H}}$ by

$$
\overline{A_{H}}=\overline{T_{H}}+K, \quad D\left(\overline{A_{H}}\right)=D\left(\overline{T_{H}}\right) \text {. }
$$

Because

$$
\begin{aligned}
(\lambda I & \left.-\overline{T_{H}}-K\right)^{-1} \\
& =\sum_{n \geq 1}\left[\left(\lambda I-\overline{T_{H}}\right)^{-1} K\right]^{n}\left(\lambda I-\overline{T_{H}}\right),
\end{aligned}
$$

therefore

$$
0 \leq\left(\lambda I-A_{H}\right)^{-1} \leq\left(\lambda I-\overline{A_{H}}\right)^{-1} .
$$


The exponential formula for strongly continuous semigroups leads to

$$
0 \leq V_{H}(t) \leq \overline{V_{H}(t)},
$$

where $V_{H}(t)_{t \geq 0}$ and $\overline{V_{H}(t)_{t \geq 0}}$ denote the strongly continuous semigroup generated by $A_{H}$ and $\overline{A_{H}}$. Because of (12),

$$
\begin{aligned}
\overline{R_{n}(t)}=\int_{t_{1}+\cdots+t_{n} \leq t, t_{i} \geq 0} & \overline{U_{H}\left(t_{1}\right)} \\
& \times K \overline{U_{H}\left(t_{2}\right)} K \cdots \overline{U_{H}\left(t_{n}\right)} K \\
& \times \overline{V_{H}\left(t-t_{1} \cdots-t_{n}\right)} d t_{1} \cdots d t_{n} .
\end{aligned}
$$

As a consequence of (12)-(33)-(38)-(39), we have (28) immediately.

Lemma 2 (see [9]). Let $B$ be the generator of a strongly continuous semigroup $U(t)$ on a Banach space $X$, and denote the $K$ bounded linear operators in X. Assume that there exist $m \in N$ and $\eta>\omega(U)$ satisfying the following:

(1) $(\lambda I-B)^{-1}\left[K(\lambda I-B)^{-1}\right]^{m}$ is compact for all $\lambda$ such that $\operatorname{Re} \lambda \geq \eta$;

(2) for every $\lambda$ satisfying $\{\lambda \in \mathrm{C} \mid \operatorname{Re} \lambda \geq \eta\}$, then

$$
\lim _{|\operatorname{Im} \lambda| \rightarrow+\infty}|\operatorname{Im} \lambda|\left\|(\lambda I-B)^{-1}\left[K(\lambda I-B)^{-1}\right]^{m}\right\|=0 .
$$

Then $R_{2 m+1}(t)$ is compact on $X$ for each $t>0$.

Lemma 3 (see [12]). Let $(\Omega, \Sigma, \mu)$ be a positive measure space and let $S, T$ be bounded linear operators on $(\Omega, \mu)$. If $T$ is compact and $0 \leq S \leq T$, then $S$ is compact.

\section{Main Result}

In this section we are ready to prove the main result of this paper. Let

$$
\Gamma_{\omega}=\left\{\lambda \in \mathbf{C}, \operatorname{Re} \lambda \geq \lambda_{0}+\omega\right\} \quad(\omega>0) .
$$

Theorem 4. If assumptions $O_{1}$ and $O_{2}$ are satisfied, for $r \in$ $[0,1)$, then

$$
\lim _{|\mathrm{Im} \lambda| \rightarrow+\infty}|\operatorname{Im} \lambda|^{r}\left\|K\left(\lambda I-T_{H}\right)^{-1} K\right\|=0,
$$

uniformly on $\Gamma_{\omega}$.

Proof. Now we are going to divide the proof into several steps.

Step One. For any $\lambda \in \Gamma_{\omega}$, prove that

$$
\lim _{|\operatorname{Im} \lambda| \rightarrow+\infty}|\operatorname{Im} \lambda|^{r}\left\|K \overline{E_{\lambda}} K\right\|=0,
$$

uniformly on $\Gamma_{\omega}$. For all $\varphi \in X_{p}$,

$$
\overline{E_{\lambda}} \varphi(\mu, v)=\frac{1}{v} \int_{0}^{\mu} e^{-\left(\left(\lambda+\sigma_{0}\right) / v\right)\left(\mu-\mu^{\prime}\right)} \varphi\left(\mu^{\prime}, v\right) d \mu^{\prime} .
$$

Using the change of variable $t=\left(\mu-\mu^{\prime}\right) / \nu$, for all $\varphi \in X_{p}$, we have

$$
\overline{E_{\lambda}} \varphi(\mu, v)=\int_{0}^{+\infty} e^{-\left(\lambda+\sigma_{0}\right) t} \varphi(\mu-t v, v) \chi_{(0, \mu / v)} d t
$$

Now, we define a bounded linear operator $\overline{E_{\lambda, \varepsilon}} \varphi(\mu, v)$ by

$$
\overline{E_{\lambda, \varepsilon}} \varphi(\mu, v)=\int_{\varepsilon}^{1 / \varepsilon} e^{-\left(\lambda+\sigma_{0}\right) t} \varphi(\mu-t v, v) \chi_{(0, \mu / v)} d t
$$

For all $\varphi \in X_{p}$, clearly, $\left(\overline{E_{\lambda, \varepsilon}}\right)_{\varepsilon>0}$ converges in the operator topology to $\overline{E_{\lambda}}$ uniformly on $\Gamma_{\omega}$ as $\varepsilon \rightarrow 0$. So, it suffices to show that, for $\varepsilon>0$,

$$
\lim _{|\operatorname{Im} \lambda| \rightarrow+\infty}|\operatorname{Im} \lambda|^{r}\left\|K \overline{E_{\lambda, \varepsilon}} K\right\|=0
$$

uniformly on $\Gamma_{\omega}$. Putting

$$
s=\mu-t v^{\prime}, \quad w(\cdot)=f(\cdot) g(\cdot),
$$

we get

$$
\begin{aligned}
& K \overline{E_{\lambda, \varepsilon}} K \varphi(\mu, v) \\
& =\alpha(\mu) f(v) \int_{0}^{a} d s \int_{\varepsilon}^{1 / \varepsilon} \frac{d t}{t} \\
& \quad \times e^{-\left(\lambda+\sigma_{0}\right) t} w\left(\frac{\mu-s}{t}\right) \alpha(s) \\
& \quad \times \int_{0}^{c} d v^{\prime \prime} g\left(v^{\prime \prime}\right) \varphi\left(s, v^{\prime \prime}\right) \chi_{(\mu-t c, \mu)}(s) .
\end{aligned}
$$

Let $K \overline{E_{\lambda, \varepsilon}} K=E_{1} \cdot E_{\varepsilon} \cdot E_{2}$, where

$$
\begin{gathered}
E_{1}: L_{p}[(0, a)(0, a)] \longrightarrow X_{p}, \\
E_{1} \varphi(\mu, v)=\alpha(\mu) f(v) \int_{0}^{a} \varphi(s, v) d s, \\
E_{2}: X_{p} \longrightarrow L_{p}(0, a), \\
E_{2} \varphi(\mu)=\alpha(\mu) f(v) \int_{0}^{c} g(v) \varphi(\mu, v) d v, \\
E_{\varepsilon}: X_{p} \longrightarrow X_{p}, \\
\left(E_{\varepsilon} \varphi\right)(\mu, s)=\int_{\varepsilon}^{1 / \varepsilon} \frac{d t}{t} e^{-\left(\lambda+\sigma_{0}\right) t} \varphi(s) \\
\times w\left(\frac{\mu-s}{t}\right) \chi_{(\mu-t c, \mu)}(s) .
\end{gathered}
$$

Since $E_{1}$ and $E_{2}$ are bounded operators, it suffices to prove that

$$
\lim _{|\operatorname{Im} \lambda| \rightarrow+\infty}|\operatorname{Im} \lambda|^{p r}\left\|E_{\varepsilon}\right\|^{p}=0
$$


uniformly on $\Gamma_{\omega}$. For all $\varphi \in L_{p}(0, a)$,

$$
\begin{aligned}
& \left\|E_{\varepsilon} \varphi\right\|^{p} \\
& =\int_{0}^{a} d \mu \mid \int_{0}^{a} d s \int_{\varepsilon}^{1 / \varepsilon} \frac{d t}{t} e^{-\left(\lambda+\sigma_{0}\right) t} \\
& \quad \times\left. w\left(\frac{\mu-s}{t}\right) \varphi(s) \chi_{(\mu-t c, \mu)}(s)\right|^{p} .
\end{aligned}
$$

Applying the Hölder inequality we get

$$
\begin{aligned}
& \mid \int_{0}^{a} d s \int_{\varepsilon}^{1 / \varepsilon} \frac{d t}{t} e^{-\left(\lambda+\sigma_{0}\right) t} \\
& \quad \times\left. w\left(\frac{\mu-s}{t}\right) \varphi(s) \chi_{(\mu-t c, \mu)}(s)\right|^{p} \\
& \leq\left(\int_{0}^{a} d s \mid \int_{\varepsilon}^{1 / \varepsilon} \frac{d t}{t} \exp \left(-\left(\lambda+\sigma_{0}\right) t\right)\right. \\
& \times \int_{0}^{a}|\varphi(s)|^{p} d s \\
& =a^{p / q} \mid \int_{\varepsilon}^{1 / \varepsilon} \frac{d t}{t} e^{-\left(\lambda+\sigma_{0}\right) t} \\
& \left.\times\left. w\left(\frac{\mu-s}{t}\right) \chi_{(\mu-t c, \mu)}\right|^{q}\right)^{p / q} \\
& \times\left. w \chi_{(\mu-t c, \mu)}\right|^{p} \cdot\|\varphi\|^{p} .
\end{aligned}
$$

Then we have

$$
\begin{aligned}
\left\|E_{\varepsilon}\right\|^{p} \leq a^{p / q} \int_{0}^{a} \mid & \int_{\varepsilon}^{1 / \varepsilon} \frac{d t}{t} e^{-\left(\lambda+\sigma_{0}\right) t} \\
& \times\left. w\left(\frac{\mu-s}{t}\right) \chi_{(\mu-t c, \mu)}(s)\right|^{p} d \mu .
\end{aligned}
$$

Putting $\mu=\mu-s, w(\cdot) \subset[\delta, c]$, and $\delta>0$, we get

$$
\begin{aligned}
& \int_{0}^{a} \mid \int_{\varepsilon}^{1 / \varepsilon} \frac{d t}{t} e^{-\left(\lambda+\sigma_{0}\right) t} \\
& \quad \times\left. w\left(\frac{\mu-s}{t}\right) \chi_{(\mu-t c, \mu)}(s)\right|^{p} d \mu \\
& \leq \int_{\varepsilon \delta}^{c / \varepsilon} \mid \int_{\varepsilon}^{1 / \varepsilon} \frac{d t}{t} e^{-\left(\lambda+\sigma_{0}\right) t} \\
& \quad \times\left. w\left(\frac{\mu}{t}\right) \chi_{(\mu-t c, \mu)}(0)\right|^{p} d \mu,
\end{aligned}
$$

so we have

$$
\begin{aligned}
\left\|E_{\varepsilon}\right\|^{p} \leq a^{p / q} \int_{\varepsilon \delta}^{c / \varepsilon} \mid & \int_{\varepsilon}^{1 / \varepsilon} \frac{d t}{t} \\
& \times\left. e^{-\left(\lambda+\sigma_{0}\right) t} w\left(\frac{\mu}{t}\right) \chi_{(\mu-t c, \mu)}(0)\right|^{p} d \mu .
\end{aligned}
$$

For any $t \leq 1 / \varepsilon$,

$$
\chi_{(\mu-t c, \mu)}(0)=\chi_{(\mu / c, 1 / \varepsilon)}(t),
$$

and we get

$$
\begin{aligned}
& \left\|E_{\varepsilon}\right\|^{p} \leq a^{p / q} \int_{\varepsilon \delta}^{c / \varepsilon} \mid \int_{\varepsilon}^{1 / \varepsilon} \frac{d t}{t} \\
& \times\left. e^{-\left(\lambda+\sigma_{0}\right) t} w\left(\frac{\mu}{t}\right) \chi_{(\mu / c, 1 / \varepsilon)}(t)\right|^{p} d \mu .
\end{aligned}
$$

So, it remains to show that

$$
\begin{aligned}
\lim _{|\operatorname{Im} \lambda| \rightarrow+\infty}|\operatorname{Im} \lambda|^{p r} \int_{\varepsilon \delta}^{c / \varepsilon} & \mid \int_{\varepsilon}^{1 / \varepsilon} \frac{d t}{t} \\
& \times\left. e^{-\left(\lambda+\sigma_{0}\right) t} w\left(\frac{\mu}{t}\right) \chi_{(\mu / c, 1 / \varepsilon)}(t)\right|^{p} d \mu=0,
\end{aligned}
$$

uniformly on $\Gamma_{\omega}$. We define now the functions

$$
\begin{gathered}
a(\cdot):\left(\varepsilon, \frac{1}{\varepsilon}\right) \longrightarrow\left(\varepsilon, \frac{1}{\varepsilon}\right), \quad \varepsilon \in(0,1) ; \\
t \longrightarrow \frac{1}{t}, \quad t \in\left(\varepsilon, \frac{1}{\varepsilon}\right), \\
\ell(\cdot)=\sum_{j=0}^{m} d_{j} \chi_{\left(a_{j}, b_{j}\right)},
\end{gathered}
$$

such that $a(t) \leq \ell(\cdot), d_{j} \geq 0,\left(a_{j}, b_{j}\right) \subset[\delta, c]$, and $0 \leq j \leq m$. So, it suffices to establish that

$$
\begin{aligned}
\lim _{|\mathrm{Im} \lambda| \rightarrow+\infty}|\operatorname{Im} \lambda|^{p r} \int_{\varepsilon \delta}^{c / \varepsilon} \mid & \int_{\varepsilon}^{1 / \varepsilon} \frac{d t}{t} \\
& \times e^{-\left(\lambda+\sigma_{0}\right) t} \ell(t) w\left(\frac{\mu}{t}\right) \\
& \times\left.\chi_{(\mu / c, 1 / \varepsilon)}(t)\right|^{p} d \mu=0
\end{aligned}
$$

uniformly on $\Gamma_{\omega}$. Because the functions $\ell(\cdot)$ and $w(\cdot)$ are simple functions, we fix $\mu \in(\varepsilon \delta, c / \varepsilon)$, and for all $t \in(\varepsilon, 1 / \varepsilon)$, we have

$$
\begin{aligned}
\ell(t) & w\left(\frac{\mu}{t}\right) \chi_{(\mu / c, 1 / \varepsilon)}(t) \\
= & \left(\sum_{j=0}^{m} d_{j} \chi_{\left(a_{j}, b_{j}\right)}(t)\right)\left(\sum_{i=0}^{n} c_{j} \chi_{\left(\mu / a_{i}, \mu / b_{i}\right)}(t)\right) \chi_{(\mu / c, 1 / \varepsilon)}(t) \\
= & \sum_{k=0}^{N(\mu, n, m)} e_{k} \chi_{\left(a_{k}(\mu), b_{k}(\mu)\right)}(t)
\end{aligned}
$$


where $N(\mu, n, m) \leq m n, \mu \in(\varepsilon \delta, c / \varepsilon), e_{k}$ is nonnegative constant and $\left(a_{k}(\mu), b_{k}(\mu)\right) \subset(\varepsilon, 1 / \varepsilon)$. We get

$$
\begin{aligned}
\int_{\varepsilon}^{1 / \varepsilon} & \frac{d t}{t} e^{-\left(\lambda+\sigma_{0}\right) t} \ell(t) w\left(\frac{\mu}{t}\right) \chi_{(\mu / c, 1 / \varepsilon)}(t) \\
= & \sum_{k=0}^{N(\mu, n, m)} e_{k} \int_{a_{k}(\mu)}^{b_{k}(\mu)} e^{-\left(\lambda+\sigma_{0}\right) t} d t \\
& =\sum_{k=0}^{N(\mu, n, m)} \frac{e_{k}}{\lambda+\sigma_{0}} \Xi(\lambda, \mu)
\end{aligned}
$$

where

$$
\Xi(\lambda, \mu)=e^{-\left(\lambda+\sigma_{0}\right) a_{k}(\mu)}-e^{-\left(\lambda+\sigma_{0}\right) b_{k}(\mu)} .
$$

This implies that

$$
\begin{aligned}
& \left|\int_{\varepsilon}^{1 / \varepsilon} \frac{d t}{t} e^{-\left(\lambda+\sigma_{0}\right) t} \ell(t) w\left(\frac{\mu}{t}\right) \chi_{(\mu / c, 1 / \varepsilon)}(t)\right| \\
& \quad \leq \frac{2}{|\operatorname{Im} \lambda|} \sum_{k=0}^{N(\mu, n, m)}\left|e_{k}\right| \\
& \quad \leq \frac{2(m n+1) \sup \|w(\cdot)\|}{\varepsilon|\operatorname{Im} \lambda|} .
\end{aligned}
$$

Consequently, we have

$$
\begin{aligned}
& \int_{\varepsilon \delta}^{c / \varepsilon}\left|\int_{\varepsilon}^{1 / \varepsilon} \frac{d t}{t} e^{-\left(\lambda+\sigma_{0}\right) t} w\left(\frac{\mu}{t}\right) \chi_{(\mu / c, 1 / \varepsilon)}(t)\right|^{p} d \mu \\
& \quad \times|\operatorname{Im} \lambda|^{p r} \leq \frac{(\Upsilon(t, \mu, m, n))^{p}}{\varepsilon^{p}|\operatorname{Im} \lambda|^{p(1-r)}}
\end{aligned}
$$

where

$$
\Upsilon(t, \mu, m, n)=2(m n+1) \sup \|w(\cdot)\| .
$$

Finally, we get

$$
\begin{aligned}
& \lim _{|\operatorname{Im} \lambda| \rightarrow+\infty}|\operatorname{Im} \lambda|^{p r} \int_{\varepsilon \delta}^{c / \varepsilon} \\
& \quad \times \mid \int_{\varepsilon}^{1 / \varepsilon} \frac{d t}{t} e^{-\left(\lambda+\sigma_{0}\right) t} w \\
& \quad \times\left.\left(\frac{\mu}{t}\right) \chi_{(\mu / c, 1 / \varepsilon)}(t)\right|^{p} d \mu \\
& \leq \lim _{|\operatorname{Im} \lambda| \rightarrow+\infty} \frac{(\Upsilon(t, \mu, m, n))^{p}}{\varepsilon^{p}|\operatorname{Im} \lambda|^{p(1-r)}}=0 .
\end{aligned}
$$

So we can get (43) immediately.

Step Two. For any $k \in \mathbf{N}$, for $r \in[0,1)$, prove that

$$
\lim _{|\operatorname{Im} \lambda| \rightarrow+\infty}|\operatorname{Im} \lambda|^{r}\left\|H_{2}\left(\overline{P_{\lambda}} H_{1}\right)^{k} \overline{D_{\lambda}} K\right\|=0,
$$

uniformly on $\Gamma_{\omega}$. Since $\mathrm{H}_{2}$ is positive and compact operator, so it suffices to establish the result for a positive operator of rank one; that is,

$$
\begin{gathered}
H_{2}: \varphi(a, v) \longrightarrow H_{2} \varphi(0, v), \\
H_{2} \varphi(0, v)=\eta(v) \int_{0}^{c} \beta(v) \varphi\left(0, v^{\prime}\right) v^{\prime} d v^{\prime},
\end{gathered}
$$

where $\eta(\cdot) \in L_{p}[0, c], \beta(\cdot) \in L_{q}[0, c]$, and $(1 / p)+(1 / q)=1$. So for any $\varphi \in X_{p}, f(\cdot) \subset[\delta, c]$, we get

$$
\begin{aligned}
& \left(H_{2}\left(\overline{P_{\lambda}} H_{1}\right)^{k} \overline{D_{\lambda}} K \varphi\right)(0, v) \\
& =\eta(v) \int_{\delta}^{c} f\left(v^{\prime}\right) d v^{\prime} e^{-\left(\lambda+\sigma_{0}\right)\left(a(k+1)-\mu^{\prime}\right) / v^{\prime}} \\
& \quad \times \int_{0}^{a} d \mu \alpha(\mu) \int_{0}^{c} d v^{\prime \prime} g\left(v^{\prime \prime}\right) \varphi\left(\mu, v^{\prime \prime}\right) .
\end{aligned}
$$

Let

$$
\left(H_{2}\left(\overline{P_{\lambda}} H_{1}\right)^{k} \overline{D_{\lambda}} K \varphi\right)(0, v)=F_{1} \cdot F_{\lambda, a} \cdot F_{2},
$$

where

$$
\begin{gathered}
F_{1}: \gamma \in \mathbf{C} \longrightarrow \gamma \eta(\cdot) \in X_{p}^{0}, \\
F_{2}: \varphi \longrightarrow \alpha(\mu) \int_{0}^{c} g(v) \varphi(\mu, v) d v \in L_{p}[(0, a), d \mu] \\
F_{\lambda, a}: L_{p}[(0, a), d \mu] \longrightarrow \mathbf{C} \\
\Phi(\cdot)=\beta(\cdot) f(\cdot), \\
\left(F_{\lambda, a} \varphi\right)(\mu, v)=\int_{0}^{a} d \mu \varphi(\mu) \int_{\delta}^{c} d v \Phi(v) \\
\\
\times e^{-\left(\lambda+\sigma_{0}\right)(a(k+1)-\mu) / v}
\end{gathered}
$$

Since $F_{1}$ and $F_{2}$ are bounded operators,

$$
\left\|H_{2}\left(\overline{P_{\lambda}} H_{1}\right)^{k} \overline{D_{\lambda}} K\right\| \leq\left\|F_{1}\right\| \cdot\left\|F_{\lambda, a}\right\| \cdot\left\|F_{2}\right\|,
$$

so it suffices to prove that

$$
\lim _{|\operatorname{Im} \lambda| \rightarrow+\infty}|\operatorname{Im} \lambda|^{p r}\left\|F_{\lambda, a}\right\|^{p}=0,
$$

uniformly on $\Gamma_{\omega}$. For any $\theta \in[a, a+\zeta], \zeta>0$, let

$$
\Theta_{\theta, \mu^{\prime}}=k a+\theta-\mu^{\prime} ;
$$

we defined a bounded linear operator $F_{\lambda, \theta}$ by

$$
\begin{gathered}
F_{\lambda, \theta}: L_{p}[(0, a), d \mu] \longrightarrow \mathbf{C}, \\
F_{\lambda, \theta} \varphi=\int_{0}^{a} d \mu^{\prime} \varphi\left(\mu^{\prime}\right) \int_{\delta}^{c} \Phi(v) \\
\times \frac{e^{-\left(\lambda+\sigma_{0}\right) \Theta_{\theta, \mu^{\prime}}}}{v} d v .
\end{gathered}
$$


Let $v \rightarrow \zeta=1 / v$ and $\varphi \in L_{p}(-a, a)$; then

$$
\begin{aligned}
F_{\lambda, \theta} \varphi=\int_{0}^{a} d \mu^{\prime} \varphi\left(\mu^{\prime}\right) & \int_{1 / c}^{1 / \delta} \widetilde{\Phi(\zeta)} \\
& \times e^{-\left(\lambda+\sigma_{0}\right) \Theta_{\theta, \mu^{\prime}}} d \varsigma,
\end{aligned}
$$

where $\widetilde{\Phi(\zeta)} \in L_{p}(1 / c, 1 / \delta)$ and

$$
\widetilde{\Phi(\zeta)}=\frac{\Phi(1 / \varsigma)}{\zeta^{2}}, \quad \varsigma \in(\delta, c)
$$

So, we can assume that

$$
\widetilde{\Phi(\cdot)}=\sum_{i=0}^{n} q_{k} \chi_{\left(\alpha_{k}, \beta_{k}\right)}(\cdot),
$$

where $q_{k} \in\left(\alpha_{k}, \beta_{k}\right)$. Clearly, using the Lebesgue dominated convergence theorem, we get the nets of operators $\left(F_{\lambda, \theta}\right)_{\theta>\alpha} \rightarrow F_{\lambda, a}\left(\theta \rightarrow a^{+}\right)$. And for all $\lambda \in \Gamma_{\omega}$, it suffices to prove that

$$
\lim _{|\operatorname{Im} \lambda| \rightarrow+\infty}|\operatorname{Im} \lambda|^{p r}\left\|F_{\lambda, \theta}\right\|^{p}=0,
$$

uniformly on $\Gamma_{\omega}$. Because

$$
\widetilde{\Phi(\varsigma)}=\sum_{i=0}^{n} q_{k} \chi_{\left(\alpha_{k}, \beta_{k}\right)}(\varsigma),
$$

we get

$$
\begin{aligned}
\int_{1 / c}^{1 / \delta} \widetilde{\Phi(\zeta)} e^{-\left(\lambda+\sigma_{0}\right) \varsigma \Theta_{\theta, \mu^{\prime}}} d \varsigma \\
=\sum_{i=0}^{n} \frac{q_{k}}{-\left(\lambda+\sigma_{0}\right) \Theta_{\theta, \mu^{\prime}}} \\
\quad \times\left(e^{-\left(\lambda+\sigma_{0}\right) \alpha_{k} \Theta_{\theta, \mu^{\prime}}}-e^{-\left(\lambda+\sigma_{0}\right) \beta_{k} \Theta_{\theta, \mu^{\prime}}}\right) .
\end{aligned}
$$

And we get

$$
\left|\int_{1 / c}^{1 / \delta} \widetilde{\Phi(\zeta)} e^{-\left(\lambda+\sigma_{0}\right) \varsigma \Theta_{\theta, \mu^{\prime}}} d \varsigma\right| \leq \frac{2(n+1)}{(\theta-a)|\operatorname{Im} \lambda|} .
$$

Applying the Hölder inequality we get

$$
\left\|F_{\lambda, \theta} \varphi\right\|^{p} \leq a^{p / q}\left|\int_{1 / c}^{1 / \delta} \widetilde{\Phi(\zeta)} e^{-\left(\lambda+\sigma_{0}\right) \varsigma \Theta_{\theta, \mu^{\prime}}} d \varsigma\right|^{p}\|\varphi\|^{p} .
$$

And we have

$$
\begin{aligned}
& \lim _{|\mathrm{Im} \lambda| \rightarrow+\infty}|\operatorname{Im} \lambda|^{p r}\left\|F_{\lambda, \theta}\right\|^{p} \\
& \quad \leq \lim _{|\operatorname{Im} \lambda| \rightarrow+\infty} \frac{a^{p / q}(2 n+2)^{p}}{(\theta-a)^{p}|\operatorname{Im} \lambda|^{p(1-r)}}=0 .
\end{aligned}
$$

So we can get (69) immediately.

Step Three. For any $N \in \mathbf{N}$, for $r \in[0,1)$, prove that

$$
\lim _{|\operatorname{Im} \lambda| \rightarrow+\infty}|\operatorname{Im} \lambda|^{r}\left\|K \overline{Q_{\lambda}} H_{1}\left(\overline{P_{\lambda}} H_{1}\right)^{N} K\right\|=0,
$$

uniformly on $\Gamma_{\omega}$. For all $\varphi \in X_{p}$, easy calculations show that

$$
\begin{aligned}
K \overline{Q_{\lambda}} H_{1}\left(\overline{P_{\lambda}} H_{1}\right)^{N} \overline{D_{\lambda}} K \varphi(\mu, v) \\
=\alpha(\mu) f(v) \int_{\delta}^{c} \frac{w\left(v^{\prime}\right)}{v^{\prime}} d v^{\prime} e^{-\left(\lambda+\sigma_{0}\right)(n a+\mu) / v^{\prime}} \\
\quad \times \int_{0}^{a} d \mu^{\prime} \alpha\left(\mu^{\prime}\right) e^{-\left(\lambda+\sigma_{0}\right)\left(a-\mu^{\prime}\right) / v^{\prime}} \\
\times \int_{\delta}^{c} d v^{\prime \prime} g\left(v^{\prime \prime}\right) \varphi\left(\mu^{\prime}, v^{\prime \prime}\right) .
\end{aligned}
$$

Let

$$
K \overline{Q_{\lambda}} H_{1}\left(\overline{P_{\lambda}} H_{1}\right)^{N} \overline{D_{\lambda}} K=G_{1} \cdot G_{\lambda, a} \cdot G_{2},
$$

where

$$
\begin{gathered}
G_{1}: \varphi \longrightarrow \alpha(\mu) f(v) \varphi(\mu) \in X_{p}, \\
G_{2}: \varphi \longrightarrow \alpha(\mu) \int_{0}^{c} g(v) \varphi(\mu, v) d v \in L_{p}[(0, a), d \mu], \\
G_{\lambda, a}: L_{p}[(0, a), d \mu] \longrightarrow L_{p}[(0, a), d \mu], \\
\left(G_{\lambda, a} \varphi\right)(\mu, v)=\int_{0}^{a} d \mu^{\prime} \varphi\left(\mu^{\prime}\right) \int_{\delta}^{c} \frac{w(v)}{v} \\
\times e^{-\left(\lambda+\sigma_{0}\right)\left((n+1) a+\mu-\mu^{\prime}\right) / v} d v .
\end{gathered}
$$

Because $G_{1}$ and $G_{2}$ are bounded operators, it suffices to prove that

$$
\lim _{|\operatorname{Im} \lambda| \rightarrow+\infty}|\operatorname{Im} \lambda|^{p r}\left\|G_{\lambda, a}\right\|=0,
$$

uniformly on $\Gamma_{\omega}$. In the same principle as (75), we can get (87) immediately.

Step Four. For any $\operatorname{Re} \lambda>\lambda_{0}$, because of (30) and assumption $\mathrm{O}_{2}$, we have

$$
\begin{gathered}
\left\|\left(\lambda-T_{H}\right)^{-1}\right\| \leq\left\|\left(\lambda-\overline{T_{H}}\right)^{-1}\right\|, \\
\left\|K\left(\lambda-T_{H}\right)^{-1} K\right\| \leq\left\|K\left(\lambda-\overline{T_{H}}\right)^{-1} K\right\| .
\end{gathered}
$$

According to (25)-(27)-(29)-(30)-(43), it suffices to establish that, for $r \in[0,1)$, prove that

$$
\lim _{|\operatorname{Im} \lambda| \rightarrow+\infty}|\operatorname{Im} \lambda|^{r}\left\|K \overline{Q_{\lambda}} H\left(\overline{P_{\lambda}} H\right)^{N} \overline{D_{\lambda}} K\right\|=0,
$$

uniformly on $\Gamma_{\omega}$. Note that $\overline{P_{\lambda}} H_{1}$ and $\overline{P_{\lambda}} H_{2}$ do not commute, so we have

$$
\left(\overline{P_{\lambda}} H\right)^{n}=\sum_{j=1}^{2^{n}} P_{j}
$$


where each $P_{j}$ is the product of $\mathrm{n}$ factors involving both $\overline{P_{\lambda}} H_{1}$ and $\overline{P_{\lambda}} H_{2}$ except the term

$$
P_{2^{n}}=\left(\overline{P_{\lambda}} H_{1}\right)^{n} .
$$

So, for $j \in\left\{1,2, \ldots, 2^{n}-1\right\}$, the operator $H_{2}$ appears at least one time in the expression of $P_{j}$. So, there exists $k \in\{1,2, \ldots$, $\left.2^{n}-1\right\}$, such that

$$
P_{j}=Q_{j} \overline{P_{\lambda}} H_{2}\left(\overline{P_{\lambda}} H_{1}\right)^{k}
$$

where $Q_{j}$ is uniformly bounded on $\Gamma_{\varepsilon}$. Now using the inequality

$$
\begin{aligned}
& \left\|K \overline{Q_{\lambda}} H P_{j} \overline{D_{\lambda}} K\right\| \leq\left\|K \overline{Q_{\lambda}} H\right\| \\
& \quad \times\left\|Q_{j} \overline{P_{\lambda}}\right\| \cdot\left\|H_{2}\left(\overline{P_{\lambda}} H_{1}\right)^{n} \overline{D_{\lambda}} K\right\|
\end{aligned}
$$

and (53), for $j \in\left\{1,2, \ldots, 2^{n}-1\right\}, r \in[0,1)$, we get

$$
\lim _{|\operatorname{Im} \lambda| \rightarrow+\infty}|\operatorname{Im} \lambda|^{r}\left\|K \overline{Q_{\lambda}} H P_{j} \overline{D_{\lambda}} K\right\|=0,
$$

uniformly on $\Gamma_{\omega}$. On the other hand, for $j=2^{n}$, we have

$$
\begin{aligned}
& \left\|K \overline{Q_{\lambda}} H P_{2^{n}} \overline{D_{\lambda}} K\right\| \\
& \leq\left\|K \overline{Q_{\lambda}} H_{1}\left(\overline{P_{\lambda}} H_{1}\right)^{n} \overline{D_{\lambda}} K\right\| \\
& \quad+\left\|K \overline{Q_{\lambda}} H_{2}\left(\overline{P_{\lambda}} H_{1}\right)^{n} \overline{D_{\lambda}} K\right\| .
\end{aligned}
$$

Using (69) again, it remains to prove that

$$
\lim _{|\operatorname{Im} \lambda| \rightarrow+\infty}|\operatorname{Im} \lambda|^{r} \| K \overline{Q_{\lambda}} H_{1}\left(\overline{P_{\lambda}} H_{1}\right)^{n} \overline{D_{\lambda}} K=0,
$$

uniformly on $\Gamma_{\omega}$. This follows from (87). This ends the proof.

Theorem 5. If assumptions $O_{1}$ and $O_{2}$ are satisfied and $r_{\sigma}\left(P_{\lambda_{0}} H_{2}\right)>1$, then the spectrum of transport operator $A_{H}$ consists of only finite isolated eigenvalues which have finite algebraic multiplicities in trip $\Gamma_{\omega}$; furthermore a real $\lambda^{*}>\lambda_{0}$ exists such that $\lambda^{*} \in \sigma_{P}\left(A_{H}\right)$, where $\sigma_{P}\left(A_{H}\right)$ denotes point spectrum set of transport operator $A_{H}$.

Proof. Consider the following.

Step One. Prove that the spectrum of the transport operator $A_{H}$ consists of, at most, finite isolated eigenvalues which have finite algebraic multiplicities in trip $\Gamma_{\omega}$. By Lemmas $1-3$, it suffices to prove that $\overline{R_{9}(t)}$ is compact operator on $X_{p}$.

On one hand, because of hypothesis $\mathrm{O}_{2}$, we have $K\left(\lambda I-\overline{T_{H}}\right)^{-1}$ and $\left(\lambda I-\overline{T_{H}}\right)^{-1} K$ is compact operator on $X_{p}$. Let

$$
\overline{\Pi(\lambda)}=\left(\lambda I-\overline{T_{H}}\right)^{-1}\left[K\left(\lambda I-\overline{T_{H}}\right)^{-1}\right]^{4} .
$$

Since $\left(\lambda I-\overline{T_{H}}\right)^{-1}$ is bounded operator and

$$
\left(\lambda I-\overline{T_{H}}\right)^{-1} \leq \frac{1}{\operatorname{Re} \lambda-\lambda_{0}},
$$

we get that $\overline{\Pi(\lambda)}$ is compact operator on $X_{p}$.

On the other hand, because of

$$
\overline{\Pi(\lambda)} \leq\left\|\left(\lambda I-\overline{T_{H}}\right)^{-1}\right\|^{3} \cdot\left\|K\left(\lambda I-\overline{T_{H}}\right)^{-1} K\right\|^{2},
$$

thanks to (29), and for $\operatorname{Re} \geq \lambda_{0}+\omega$, we have

$$
\left(\lambda I-\overline{T_{H}}\right)^{-1} \leq \frac{1}{\omega} .
$$

So for $\operatorname{Re} \geq \lambda_{0}+\omega$, together with (102), (103), and (104) we have

$$
\begin{aligned}
& |\operatorname{Im} \lambda|\|\overline{\Pi(\lambda)}\| \\
& \quad \leq \frac{1}{\omega^{3}}\left[|\operatorname{Im} \lambda|^{1 / 2}\left\|K\left(\lambda I-\overline{T_{H}}\right)^{-1} K\right\|\right]^{2} .
\end{aligned}
$$

By Theorem 4, we get

$$
\begin{aligned}
& \lim _{|\operatorname{Im} \lambda| \rightarrow+\infty}|\operatorname{Im} \lambda|\|\overline{\Pi(\lambda)}\| \\
& \quad \leq \frac{1}{\omega^{3}}\left[\lim _{|\operatorname{Im} \lambda| \rightarrow+\infty}|\operatorname{Im} \lambda|^{1 / 2}\left\|K\left(\lambda I-\overline{T_{H}}\right)^{-1} K\right\|\right]^{2} \\
& \quad=0
\end{aligned}
$$

uniformly on $\Gamma_{\omega}$. Now, applying Lemma 3 we conclude that, for each $t>0, R_{9}(t)$ is compact operator on $X_{p}$, together with [3]; we end Step one. Now we consider again the resolvent equation which is equivalent to solving in $X_{p}^{a}$ the following one:

$$
\psi^{a}=P_{\lambda} H_{1} \psi^{a}+P_{\lambda} H_{2} \psi^{a}+E_{\lambda} \varphi .
$$

If $\lambda>\lambda_{0}$, then the operator $\left(I-P_{\lambda} H_{1}\right)^{-1}$ is bounded invertible and (104) becomes

$$
\psi^{a}=\Xi_{\lambda} \psi^{a}+L_{\lambda} \varphi
$$

where

$$
\begin{gathered}
\Xi_{\lambda}=\left(I-P_{\lambda} H_{1}\right)^{-1} P_{\lambda} H_{2}, \\
L_{\lambda}=\left(I-P_{\lambda} H_{1}\right)^{-1} E_{\lambda} .
\end{gathered}
$$

Step Two. Prove that $\left(I-\Xi_{\lambda}\right)^{-1}$ exists for $\lambda$ in the half plane

$$
\Gamma=\left\{\lambda \in \mathbf{C}, \operatorname{Re} \lambda>-\sigma_{0}\right\}
$$

with $|\operatorname{Im} \lambda|$ sufficiently large.

(i) Let $\lambda$ be an element of

$$
\Gamma_{0}=\left\{\lambda \in \mathbf{C},-\sigma_{0}<\operatorname{Re} \lambda \leq \lambda_{0}\right\},
$$


and set

$$
\begin{gathered}
P_{\lambda}^{*}: X_{q}^{a} \longrightarrow X_{q}^{0}, \\
\left(P_{\lambda}^{*} g\right)(0, v)=g(a, v) e^{-(1 / v) \int_{0}^{a}\left(\lambda+\sigma\left(\mu^{\prime}, v\right)\right) d \mu^{\prime},} \\
H_{2}^{*}: X_{q}^{a} \longrightarrow X_{q}^{0}, \\
\left(H_{2}^{*} g\right)(a, v)=\eta(v) \int_{0}^{c} \beta\left(v^{\prime}\right) g\left(0, v^{\prime}\right) d v^{\prime},
\end{gathered}
$$

where $P_{\lambda}^{*}$ and $H_{2}^{*}$ are the dual operators of $P_{\lambda}$ and $H_{2}$. Easy calculations show that the dual $\left(\mathrm{P}_{\lambda} \mathrm{H}_{2}\right)^{*}$ of the operator $\mathrm{P}_{\lambda} \mathrm{H}_{2}$ is $H_{2}^{*} P_{\lambda}^{*}$. Let

$$
\lambda_{n}=\zeta+i t_{n}
$$

be a sequence in $\Gamma$ such that

$$
\lim _{n \rightarrow+\infty}\left|t_{n}\right|=+\infty .
$$

We consider $\varphi \in X_{q}^{a}$; then (112) show that

$$
\begin{aligned}
& H_{2}^{*} P_{\lambda_{n}}^{*} \varphi=\int_{0}^{c} e^{-\left(1 / v^{\prime}\right) \int_{0}^{a}\left(\zeta+i t_{n}+\sigma\left(\mu^{\prime}, v\right)\right) d \mu^{\prime}} \\
& \times \varphi\left(a, v^{\prime}\right) \eta(v) \beta\left(v^{\prime}\right) v^{\prime} d v^{\prime} .
\end{aligned}
$$

By the Riemann-Lebesgue theorem, we obtain

$$
\lim _{n \rightarrow+\infty}\left|H_{2}^{*} P_{\lambda_{n}}^{*} \varphi(a, v)\right|=0
$$

a.e. on $\{a\} \times(0, c)$. Furthermore, for every integer $n$, we have

$$
\left|H_{2}^{*} P_{\lambda_{n}}^{*} \varphi(a, v)\right| \leq|\eta(v)| \int_{0}^{c}\left|\varphi\left(a, v^{\prime}\right)\right|\left|\beta\left(v^{\prime}\right)\right| v^{\prime} d v^{\prime} .
$$

By the dominated convergence theorem, we get

$$
\lim _{|\operatorname{Im} \lambda| \rightarrow+\infty}\left|H_{2}^{*} P_{\lambda}^{*} \varphi\right|=0,
$$

for all $\varphi \in X_{p}^{a}(\lambda \in \Gamma)$.

(ii) Prove that the family $H_{2}^{*} P_{\lambda}^{*}, \lambda \in \Gamma_{0}$, is collectively compact. Let $\Theta$ be the unit ball of the space $X_{p}^{a}$ and let $\left(x_{n}\right)$ be a sequence in $\cup_{\lambda \in \Gamma_{0}}\left(H_{2}^{*} P_{\lambda}^{*} \Theta\right)$; then there exists a sequence $\left(\varphi_{n}\right)$ in $\Theta$ such that

$$
x_{n}=P_{\lambda_{n}}^{*} \varphi_{n} .
$$

Since $H_{2}^{*}$ is compact and $\left(x_{n}\right)$ is bounded in the space $X_{p}^{a}$, there exists a converging subsequence of $\left(H_{2}^{*} x_{n}\right)$ in $\overline{\cup_{\lambda \in \Gamma_{0}}\left(H_{2}^{*} P_{\lambda}^{*} \Theta\right)}$. This is the wanted.

(iii) The use of (i) and (ii) together with Anselone's Proposition [17, Proposition 3.1] gives

$$
\lim _{|\operatorname{Im} \lambda| \rightarrow+\infty}\left\|\left(H_{2}^{*} P_{\lambda}^{*}\right)^{2}\right\|=0
$$

uniformly on $\Gamma_{0}$. Then

$$
\lim _{|\operatorname{Im} \lambda| \rightarrow+\infty} r_{\sigma}\left(H_{2}^{*} P_{\lambda}^{*}\right)=0
$$

uniformly on $\Gamma_{0}$. Since

$$
\begin{gathered}
r_{\sigma}\left(H_{2}^{*} P_{\lambda}^{*}\right) \leq\left\|\left(H_{2}^{*} P_{\lambda}^{*}\right)^{2}\right\|^{1 / 2}, \\
r_{\sigma}\left(H_{2}^{*} P_{\lambda}^{*}\right)=r_{\sigma}\left(P_{\lambda} H_{2}\right),
\end{gathered}
$$

therefore, we obtain

$$
\lim _{|\mathrm{Im} \lambda| \rightarrow+\infty} r_{\sigma}\left(P_{\lambda} H_{2}\right)=0,
$$

uniformly on $\Gamma_{0}$. This ends Step two by (iii).

Step Three. We will prove that $\sigma_{P}\left(A_{H}\right) \neq \emptyset$.

First, because of

$$
\Xi_{\lambda}=\left(I-P_{\lambda} H_{1}\right)^{-1} P_{\lambda} H_{2},
$$

there exists $\lambda_{1}>\lambda_{0}$ for $\lambda>\lambda_{1}$ and we get

$$
\left(I-P_{\lambda} H_{1}\right)^{-1} \leq\left(I-P_{\lambda_{1}} H_{1}\right)^{-1} .
$$

Hence

$$
\left(\Xi_{\lambda}\right)^{3} \leq\left(I-P_{\lambda} H_{1}\right)^{-1} P_{\lambda} H_{2}\left(\Xi_{\lambda}\right)^{2} .
$$

Because $H_{2}$ is compact on $X_{p}$ and

$$
\lim _{\lambda \rightarrow+\infty} P_{\lambda}=0,
$$

we get that $\left(P_{\lambda_{1}} H_{2}\right)^{2}$ is compact on $X_{p}$, and we have

$$
\begin{aligned}
\left\|\Xi_{\lambda}\right\|^{3} \leq & \left\|\left(I-P_{\lambda_{1}} H_{1}\right)^{-1}\right\| \cdot\left\|P_{\lambda_{1}} H_{2}\right\| \\
& \times\left\|\left(\Xi_{\lambda_{1}}\right)^{2}\right\| \longrightarrow 0 \quad(\lambda \longrightarrow+\infty) .
\end{aligned}
$$

And we get

$$
\lim _{\lambda \rightarrow+\infty} r_{\sigma}\left(\Xi_{\lambda}\right)=0 .
$$

Next, by spectral mapping theorem, we get that $\Xi_{\lambda}$ is continuous decreasing function on $\lambda$, since

$$
\lambda \in \sigma_{P}\left(A_{H}\right) \neq \emptyset \Longleftrightarrow 1 \in \sigma\left(\Xi_{\lambda}\right) .
$$

Because $\Xi_{\lambda}$ is continuous decreasing function on $\lambda$, it suffices to prove that

$$
\lambda \in \sigma_{P}\left(A_{H}\right) \neq \emptyset \Longleftrightarrow \lim _{\lambda \rightarrow \lambda_{0}} r_{\sigma}\left(\Xi_{\lambda}\right)>1 .
$$

Let

$$
\overline{\Xi_{\lambda}}=\left(I-\overline{P_{\lambda}} H_{1}\right)^{-1} \overline{P_{\lambda}} H_{2} ;
$$

we have $\overline{\Xi_{\lambda}} \rightarrow \Xi_{\lambda}\left(\lambda>\lambda_{0}\right)$, since

$$
\overline{\Xi_{\lambda}}=\sum_{n \geq 0}\left(\overline{P_{\lambda}} H_{1}\right)^{n} \overline{P_{\lambda}} H_{2} \geq \overline{P_{\lambda}} H_{2},
$$


and we get

$$
\begin{gathered}
\Xi_{\lambda} \geq P_{\lambda} H_{2}, \\
\lim _{\lambda \rightarrow \lambda_{0}} r_{\sigma}\left(\Xi_{\lambda}\right) \geq \lim _{\lambda \rightarrow \lambda_{0}} r_{\sigma}\left(P_{\lambda} H_{2}\right) .
\end{gathered}
$$

Due to the hypothesis $r_{\sigma}\left(P_{\lambda_{0}} H_{2}\right)>1$, we get

$$
\lim _{\lambda \rightarrow \lambda_{0}} r_{\sigma}\left(\Xi_{\lambda}\right)>1 \text {. }
$$

Finally, according to (135) and the fact that $\Xi_{\lambda}$ is continuous decreasing function on $\lambda$, we know that there must be a real $\lambda^{*}>\lambda_{0}$ such that

$$
r_{\sigma}\left(\Xi_{\lambda^{*}}\right)=1
$$

Hence, $\lambda^{*} \in \sigma_{P}\left(A_{H}\right) \neq \emptyset$. This ends the proof.

\section{Conflict of Interests}

The authors declare that there is no conflict of interests regarding the publication of this paper.

\section{Acknowledgments}

The authors would like to express their gratitude to the referee for his/her valuable suggestions. The research was supported by National Natural Science Foundation of China (no. 11461055), the Natural Science Foundation of Jiangxi Province of China (no. 20132BAB201002) and the Office of Education of Jiangxi Province of China (no. GJJ13706).

\section{References}

[1] M. Rotenberg, "Transport theory for growing cell populations," Journal of Theoretical Biology, vol. 103, no. 2, pp. 181-199, 1983.

[2] K. Latrach and H. Megdiche, "Spectral properties and regularity of solutions to transport equations in slab geometry," Mathematical Methods in the Applied Sciences, vol. 29, no. 17, pp. 20892121, 2006.

[3] K. Latrach and A. Dehici, "Spectral properties and time asymptotic behaviour of linear transport equations in slab geometry," Mathematical Methods in the Applied Sciences, vol. 24, no. 10, pp. 689-711, 2001.

[4] S. H. Wang, M. Z. Yang, and G. Q. Xu, "The spectrum of the transport operator with generalized boundary conditions," Transport Theory and Statistical Physics, vol. 25, no. 7, pp. 811823, 1996.

[5] M. Mokhtar-Kharroubi, "Time asymptotic behaviour and compactness in transport theory," European Journal of Mechanics B: Fluids, vol. 11, no. 1, pp. 39-68, 1992.

[6] M. Boulanouar, "A mathematical study in the theory of dynamic population," Journal of Mathematical Analysis and Applications, vol. 255, no. 1, pp. 230-259, 2001.

[7] B. Lods and M. Sbihi, "Stability of the essential spectrum for 2D-transport models with Maxwell boundary conditions," Mathematical Methods in the Applied Sciences, vol. 29, no. 5, pp. 499-523, 2006.

[8] M. Rotenberg, "Theory of distributed quiescent state in the cell cycle," Journal of Theoretical Biology, vol. 96, no. 3, pp. 495-509, 1982.
[9] K. Latrach, H. Megdiche, and M. A. Taoudi, "A compactness result for perturbed semigroups and application to a transport model," Journal of Mathematical Analysis and Applications, vol. 359, no. 1, pp. 88-94, 2009.

[10] A. Jeribi, H. Megdiche, and N. Moalla, "On a transport operator arising in growing cell populations. II. Cauchy problem," Mathematical Methods in the Applied Sciences, vol. 28, no. 2, pp. 127-145, 2005.

[11] K. Latrach and H. Megdiche, "Time asymptotic behaviour for Rotenberg's model with Maxwell boundary conditions," Discrete and Continuous Dynamical Systems A, vol. 29, no. 1, pp. 305-321, 2011.

[12] M. Boulanouar, "Transport equation in cell population dynamics II," Electronic Journal of Differential Equations, vol. 2010, 20 pages, 2010.

[13] S. H. Wang and G. F. Cheng, "Spectral problem for transport equations with a proliferating cell population," Acta Mathematica Scientia A, vol. 33, no. 1, pp. 71-77, 2013.

[14] K. Latrach and A. Zeghal, "Existence results for a boundary value problem arising in growing cell populations," Mathematical Models \& Methods in Applied Sciences, vol. 13, no. 1, pp. 1-17, 2003.

[15] B. Lods and M. Mokhtar-Kharroubi, "On the theory of a growing cell population with zero minimum cycle length," Journal of Mathematical Analysis and Applications, vol. 266, no. 1, pp. 70-99, 2002.

[16] A. Jeribi, "Time asymptotic behaviour for unbounded linear operator arising in growing cell populations," Nonlinear Analysis: Real World Applications, vol. 4, no. 5, pp. 667-688, 2003.

[17] A. P. Anselone and J. M. Palmer, "Spectral analysis of collectively compact, strongly convergent operator sequences," Pacific Journal of Mathematics, vol. 25, pp. 423-431, 1968. 


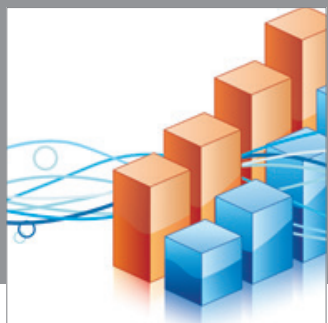

Advances in

Operations Research

mansans

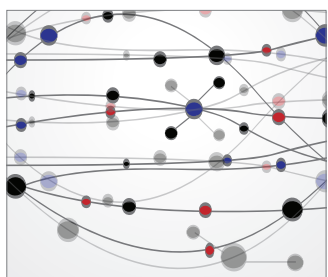

The Scientific World Journal
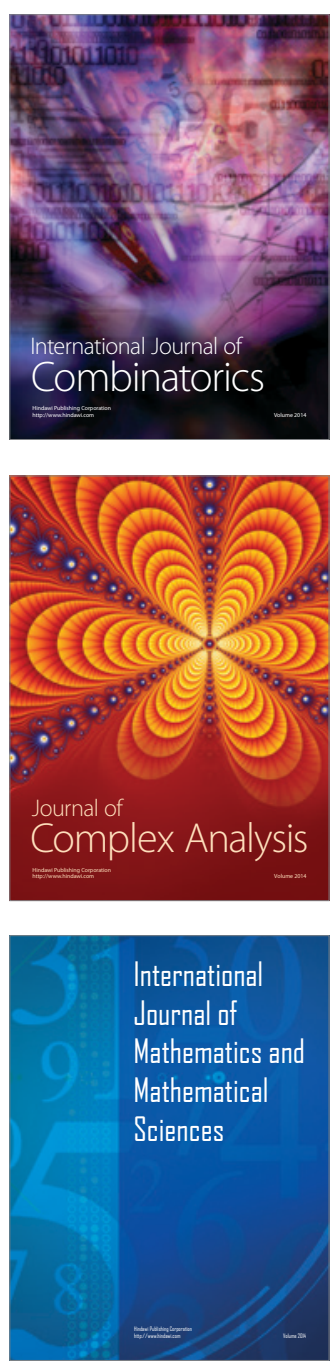
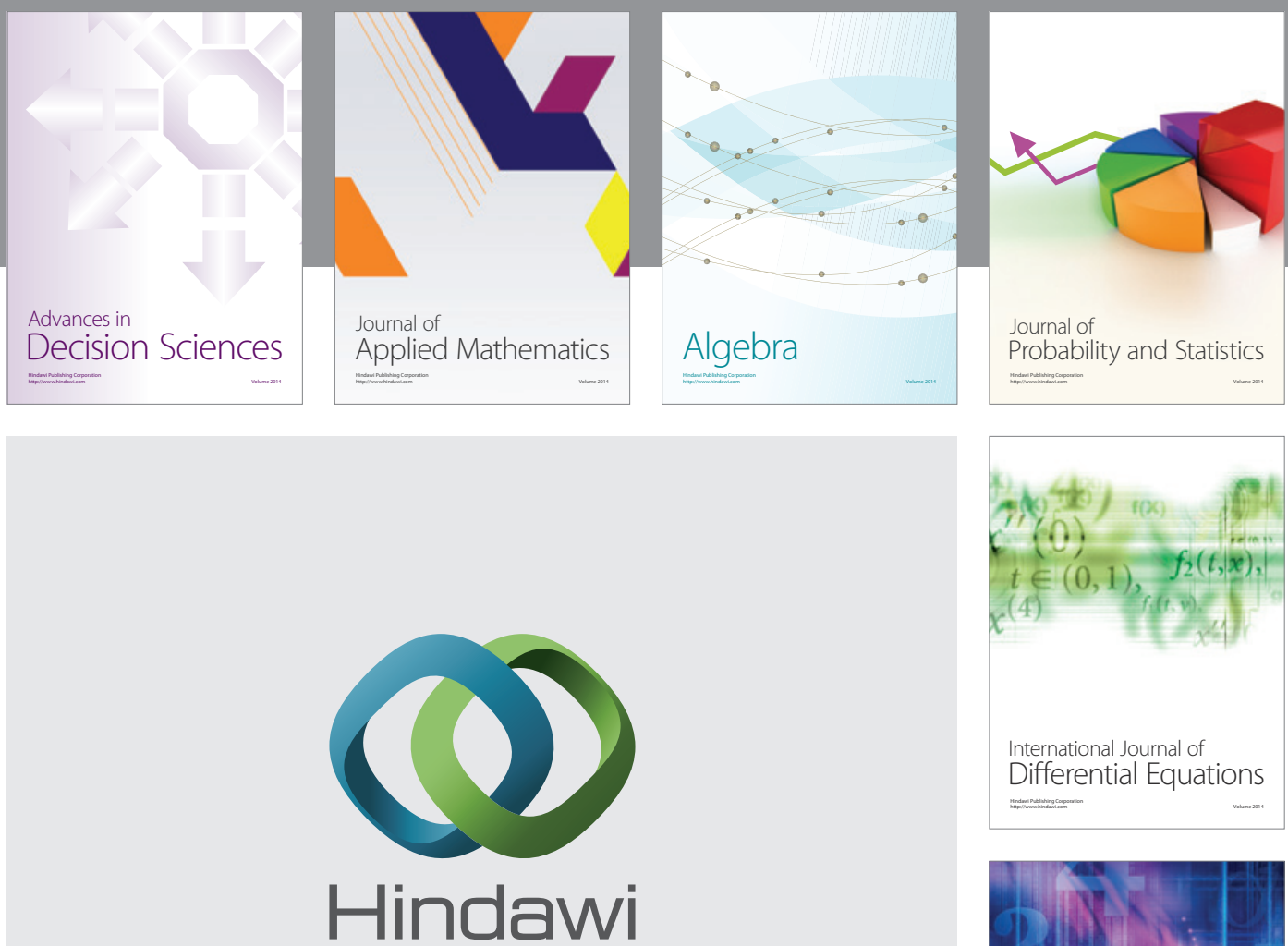

Submit your manuscripts at http://www.hindawi.com
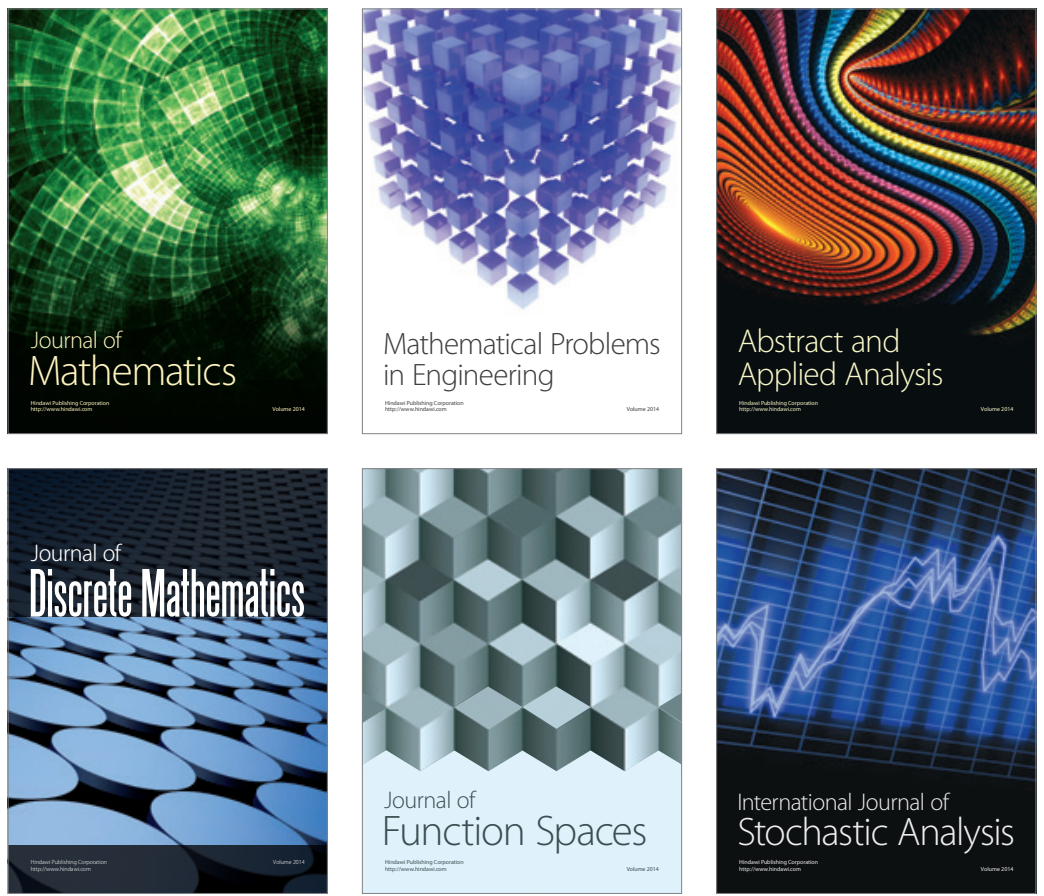

Journal of

Function Spaces

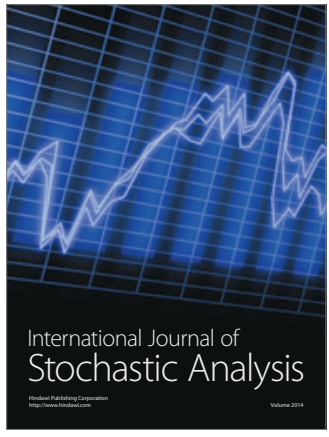

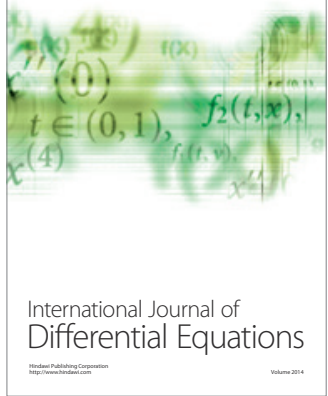
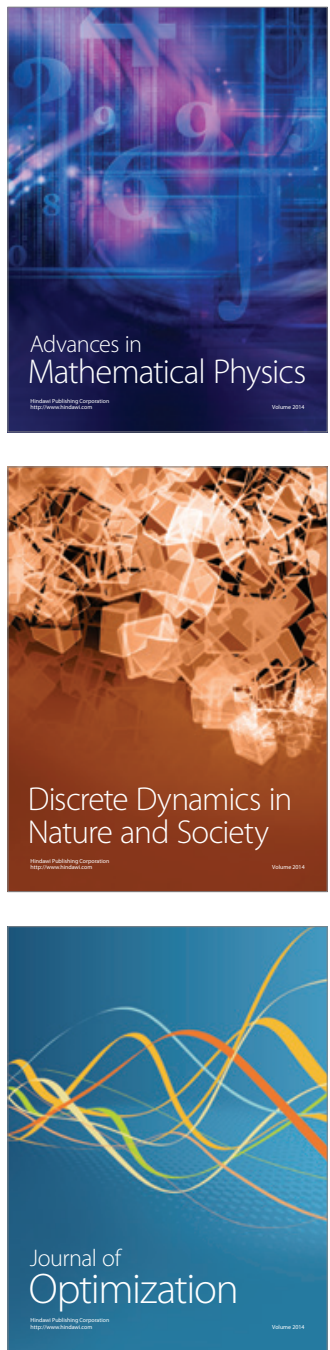